\title{
Early stem growth mutation alters metabolic flux changes enhance sesquiterpenoids biosynthesis in Atractylodes lancea (Thunb.) DC.
}

\author{
Di Wang $^{1,2} \cdot$ Fei Chen $^{1,2}\left(\mathbb{0} \cdot\right.$ Chun-Yan Wang $^{1,2} \cdot$ Xu Han $^{1,2} \cdot$ Chuan-Chao Dai $^{1}$
}

Received: 10 November 2021 / Accepted: 21 January 2022 / Published online: 1 February 2022

(c) The Author(s), under exclusive licence to Springer Nature B.V. 2022

\begin{abstract}
Atractylodes lancea (Thunb.) DC. is a well-known medicinal herb in China, containing abundant active components, including a variety of sesquiterpenoids. Owing to a shortage of wild resources, artificial cultivation has become the main breeding mode, leading to the germplasm degradation. In preliminary research, our research group found that a mutant tissue culture seedling of A. lancea is an excellent germplasm resource, characterized by early stem growth and higher sesquiterpenoid content than that of the wild type. In this study, the physiological and biochemical mechanisms underlying efficient sesquiterpenoids synthesis by this mutant $A$. lancea were systematically evaluated. The results showed that the photosynthetic efficiency, central carbon metabolism efficiency, and energy metabolism efficiency were significantly improved in mutant A. lancea compared with the wild type, and the content of endogenous hormones, such as gibberellin and jasmonic acid, changed significantly. In addition, levels of key metabolites and the expression level of key genes in the mevalonate and 2-C-methyl-D-erythritol-4-phosphate pathways were significantly higher in mutant type than in wild type, resulting in elevated sesquiterpenoid synthesis in the mutant. These physiological and biochemical properties explain the rapid growth and high sesquiterpenoid content of mutant A. lancea.
\end{abstract}

\section{Key message}

Efficient sesquiterpenoids synthesis in early stem growth mutation of Atractylodes lancea could be explained by the biological activities enhancement and up-regulation of sesquiterpene synthesis pathway via gibberellin and jasmonic acid.

Keywords Atractylodes lancea (Thunb.) DC. · Sesquiterpenoid · Primary metabolism · Plant hormone · Early stem growth mutation

\begin{tabular}{|c|c|c|c|}
\hline \multicolumn{2}{|c|}{ Abbreviations } & TCA & Tricarboxylic acid \\
\hline \multicolumn{2}{|r|}{ A. lancea Atractylodes lancea (Thunb.) DC. } & EMP & Glycolysis \\
\hline \multirow{2}{*}{\multicolumn{2}{|c|}{ GC Gas chromatography }} & MVA & Mevalonate \\
\hline & & MEP & 2-C-methyl-D-erythritol-4-phosphate \\
\hline \multirow{2}{*}{\multicolumn{2}{|c|}{ Communicated by Wagner Campos Otoni. }} & IPP & Isopentenyl diphosphate \\
\hline & & DMAPP & Dimethylallyl diphosphate \\
\hline \multirow{2}{*}{\multicolumn{2}{|c|}{$\begin{array}{l}\triangle \text { Fei Chen } \\
\text { chenfei@njnu.edu.cn }\end{array}$}} & HMGR & 3-Hydroxy-3-methylglutaryl CoA synthase \\
\hline & & DXR & 1-Deoxy-D-xylulose-5-phosphate \\
\hline \multirow{2}{*}{\multicolumn{2}{|c|}{$\begin{array}{l}\triangle \text { Chuan-Chao Dai } \\
\text { daichuanchao@njnu.edu.cn }\end{array}$}} & & reductoisomerase \\
\hline & & ELISA & Enzyme linked immunosorbent assay \\
\hline \multirow[t]{5}{*}{1} & Jiangsu Key Laboratory for Microbes and Functional & CPS & $\beta$-Caryophyllene synthase \\
\hline & Genomics, Jiangsu Engineering and Technology Research & GAS & Germacrene A synthase \\
\hline & Center for Industrialization of Microbial Resources, & NES & Nerolidol synthase \\
\hline & School of Life Sciences, Nanjing Normal University, & GA & Gibberellin \\
\hline & Nanjing 210023, China & JA & Jasmonic acid \\
\hline \multirow[t]{3}{*}{2} & Nanjing Engineering Research Center for Functional & SA & Salicylic acid \\
\hline & Components Development of Featured Biological Resources, & $\mathrm{BR}$ & Brassinolide \\
\hline & $\begin{array}{l}\text { School of Life Sciences, Nanjing Normal University, } \\
\text { Nanjing 210023, China }\end{array}$ & IAA & Indoleacetic acid \\
\hline
\end{tabular}




\section{ETH Ethylene}

CTK Cytokinin

\section{Introduction}

Atractylodes lancea (Thunb.) DC. is a perennial herb in the family Composite (Jun et al. 2018). The wild resources are mainly distributed in Jiangsu, Henan, Hubei, and other provinces in China (Xu et al. 2016). In China, the medical material of $A$. lancea has played an important role in the prevention and treatment of coronavirus disease 2019 (Liu et al. 2021). The active components of $A$. lancea are sesquiterpenoids, including atractylone, hinesol and $\beta$-eudesmol (Liu et al. 2016). These components mainly accumulate in the oil chamber of the rhizome, and are referred to as volatile oils. As a well-known traditional herb in China with excellent efficacy, demand for A. lancea is high. However, unscientific picking has sharply reduced populations of wild A. lancea, and the species is listed as an endangered protected plant in some areas of China.

Like other endangered medicinal plants, the main cultivation methods of $A$. lancea are aseptic tissue culture and artificial cultivation. Rapid propagation technology based on aseptic tissue culture has developed decades ago. Plant tissue culture could indeed yield a large number of $A$. lancea seedlings quickly without climate restrictions. However, sesquiterpenoid content in tissue culture seedlings is obviously lower than that of wild plants. Common strategies to improve the contents of active components, include the manipulation of environmental factors, such as light and water. A proper shading rate can increase plant growth, photosynthesis, biomass, and antioxidant enzyme activity (Tang et al. 2015). During the growth period, droughts and floods could have negative impacts on growth and active components, while appropriate drought and flood stress in the late fruit stage could promote rhizome development and volatile oil accumulation (Zhang et al. 2021). Appropriate biological stress in A. lancea could result in the accumulation of secondary metabolites, without affecting normal growth. Zhou et al. inoculated A. lancea with the endophytic bacteria Pseudomonas fluorescens AlEB7B, which triggered plant defense mechanisms and promoted the accumulation of sesquiterpenoids (Zhou et al. 2018). Additionally, the endophytic fungus Gilmaniella sp. A112 realizes an antagonistic balance with $A$. lancea via mannan, resulting in sesquiterpenoids accumulation via defense mechanisms (Chen et al. 2016), including jasmonic acid (JA) (Ren and Dai. 2012), salicylic acid (SA) (Ren et al. 2017), abscisic acid (ABA) (Wang et al. 2015), brassinolide (BR) (Ren and Dai. 2013) and ethylene (ETH) (Yuan et al. 2016).

Although volatile oils in $A$. lancea could be improved to a certain extent altering external abiotic and biological factors, this does not address the tissue of the degradation of A. lancea germplasm resources. Obtaining excellent germplasm resources with a high volatile oil content is a more long-term and effective method. In A. lancea, the volatile oil usually accumulates in the rhizome (Hiraoka 1995), and the rhizome develops from the stem. In our previous study, we found that the wild-type $A$. lancea tissue culture seedlings have no stem, which may be one of the reasons for the low content of volatile oil in tissue culture seedlings. Cultivating A. lancea with stem is a new breeding direction.

Rhizome formation is generally affected by nutrients, plant hormones, and regulatory factors (Guo et al. 2021). Nutrients are important for plant growth, development, and regeneration ability. In particular, sucrose is not only a raw material for plant rhizome development but can also regulate the rhythm of plant rhizome development (Bessho-Uehara et al. 2018). Plant hormones perform different functions in different plants via signaling pathways, they participate in the formation of complex and orderly networks in plants. Synergistic and antagonistic effects of plant hormones control the behavior of plants at different developmental stages (López-Bucio et al. 2003). For example, a high concentration of auxin will stimulate the production of adventitious roots (Patten and Glick. 2002), however, in gramineous plants, auxin has a negative regulatory effect on rhizome formation, while gibberellin (GA) promotes it. These hormones function together to regulate the rhizome development process in gramineous plants (Hu et al. 2011). Regulatory factors could recognize or bind to cis-acting elements in genes, and participate in the transcription efficiency of target genes involved in rhizome development (Malamy. 2005).

According to the new breeding direction, the mutant $A$. lancea tissue culture seedlings with early stem growth were successfully obtained in our lab. In this study, based on the factors determining plant rhizome formation, the advantages of mutant A. lancea were evaluated by comparisons of agronomic characters, physiological parameters, biochemical indexes, and gene expression levels between wild-type and mutant $A$. lancea. These results provide insight into the physiological and biochemical mechanisms underlying the high sesquiterpenoid content in mutant A. lancea, and lay a foundation for the synthesis of sesquiterpenoids and the optimization of $A$. lancea germplasm resources.

\section{Materials and methods}

\section{Plant tissue culture conditions}

Seedlings were collected from genuine wild plants of $A$. lancea in Maoshan area of Jiangsu Province in China ( $31^{\circ}$ $\left.47^{\prime} \mathrm{N}, 119^{\circ} 19^{\prime} \mathrm{E}\right)$. Plants were identified as authentic by Professor Chuan-Chao Dai from Nanjing Normal University, 
and some plants were transplanted in the botanical garden of Nanjing Normal University. Mutant plants of A. lancea with early stem growth were obtained by our research group, and specimens are preserved in the Rare Animals and Plants Museum of Nanjing Normal University (the preservation number of wild-type is S01240, and the mutant is S01240-1).

Plant tissue culture of A. lancea was carried out according to the method of Zhou et al. (Zhou et al. 2018). All explants were cultured in incubator, with light at $25^{\circ} \mathrm{C}$ for $12 \mathrm{~h}$ (light intensity $3400 \mathrm{~lm} \mathrm{~m}^{-2}$ ) and no light at $18^{\circ} \mathrm{C}$ for $12 \mathrm{~h}$ to simulate the alternation of day and night. In order to obtain the mutant $A$. lancea with early stem growth, 15 to $25 \mathrm{mg} \mathrm{L}^{-1} \mathrm{GA}, 200$ to $300 \mathrm{mg} \mathrm{L}^{-1} \mathrm{JA}$ and 150 to $200 \mathrm{mg}$ $\mathrm{L}^{-1} \mathrm{CTK}$ were added to the culture medium of wild-type tissue culture seedlings. After 2 weeks of plant hormone treatment, kept incubation temperature at $38^{\circ} \mathrm{C}$ for 1 week. Then cultured under conventional conditions for 2 months, some early stem growth mutations appeared. The mutant tissue culture seedlings were inserted into the differentiation medium and cultured under conventional conditions for subculture, the plantlets that still retained the mutant character in the tenth generation were identified as mutant A. lancea.

\section{Measurement of agronomic characters}

Six mutant seedlings and six wild-type seedlings of A. lancea were cultured for 1 month, 2 months, 3 months and 6 months. Fresh weight, plant height, leaf number, root length and root number were measured.

\section{Measurement of photosynthetic efficiency}

Three mutant seedlings and three wild-type seedlings of $A$. lancea were cultured for 1 month, 2 months, 3 months, and 6 months. The photosynthetic rate was measured using the LI-6400XT extractable photosynthetic rate instrument (LICOR, Lincoln, NE, USA) at 10:00-11:00 a.m., $25^{\circ} \mathrm{C}$, and $50 \%$ relative humidity. Chlorophyll was extracted by $95 \%$ ethanol, and the chlorophyll content $\left(\mathrm{mg} \mathrm{L}^{-1}\right)$ was calculated by the Arnon method (Porra. 2002). The relevant calculation formulas are as follows: $\mathrm{Chl} \mathrm{a}=12.72 \mathrm{~A}_{663}-2.59 \mathrm{~A}_{645}$, Chl $\mathrm{b}=22.88 \mathrm{~A}_{645}-4.67 \mathrm{~A}_{663}, \mathrm{Chl}(\mathrm{a}+\mathrm{b})=\mathrm{Chl} \mathrm{a}+\mathrm{Chl} \mathrm{b}$.

\section{Extraction and measurement of sesquiterpenoids}

The clean tissue culture seedlings were dried at $37{ }^{\circ} \mathrm{C}$ for $36 \mathrm{~h}$ to constant weight. After grinding to powder in a mortar, a four-fold volume of cyclohexane (W: V) was added, followed by extraction in the dark for $8 \mathrm{~h}$. The sesquiterpenoid content was detected by gas chromatography (GC). The Agilent 7890A GC (Agilent Technologies, Santa Clara, CA) with HP-5 capillary column $(30 \mathrm{~m} \times 0.32 \mathrm{~mm} \times 0.10 \mu \mathrm{m})$ and flame ionization detector (FID) were used, and samples were tested according to the following heating program: $70{ }^{\circ} \mathrm{C}$ for $1 \mathrm{~min}, 8{ }^{\circ} \mathrm{C} \mathrm{m^{-1 }}$ to $200{ }^{\circ} \mathrm{C}, 20{ }^{\circ} \mathrm{C} \mathrm{min}{ }^{-1}$ to $300{ }^{\circ} \mathrm{C}$ for $5 \mathrm{~min}$. The temperature of the sample was $240{ }^{\circ} \mathrm{C}$, the temperature of the detector was $350{ }^{\circ} \mathrm{C}$, the carrier gas was high-purity nitrogen, the flow rate was $0.92266 \mathrm{~mL} \mathrm{~min}^{-1}$, and the injection volume was $1 \mu \mathrm{L}$. Twelve kinds of sesquiterpenoids were analyzed by GC. Among them, caryophyllene oxide, cedrol, nerolidol, farnesene, $\beta$-caryophyllene and valencene were purchased from Sigma-Aldrich with purity $>99 \%$; germacrene D and zingiberene were purchased from Chengdu MUST Bio-Technology Co., Ltd with purity $>95 \%$; $\beta$-sesquiphellandrene was purchased from Toronto Research Chemicals with purity $>95 \%$; Atractylone, $\beta$-eudesmol and hinesol were isolated and purified by our research group with purity $>95 \%$.

\section{Extraction and measurement of plant hormones}

The samples of mutant seedlings and wild-type seedlings of A. lancea were cultured for 1 month, 2 months, 3 months, and 6 months. JA and SA were extracted by methanol, referring to Ren's method (Ren and Dai, 2012). The Agilent 1100 high-performance liquid chromatography system (Agilent Technologies, Santa Clara, CA) was used for detection. The chromatographic column was the Agilent HC-C18, with an inner diameter of $4.6 \mathrm{~mm}$ and a length of $250 \mathrm{~mm}$. The mobile phase flow rate was $1 \mathrm{~mL} \mathrm{~min}{ }^{-1}$, the column temperature was $25{ }^{\circ} \mathrm{C}$. The detection wavelength of JA was $217 \mathrm{~nm}$, and the detection wavelength of SA was $300 \mathrm{~nm}$. Gibberellin (GA), cytokinin (CTK) and indoleacetic acid (IAA) were extracted with methanol, and purification was performed following the methods described by Liu (Liu et al. 2011) and Yang (Yang et al. 2012). Ethylene (ETH) was extracted with precooled phosphoric acid buffer $(\mathrm{pH}$ 7.4), referring to the extraction method of Zhou (Zhou et al. 2016). GA content was measured using the Plant GA ELISA Kit (Jingmei Biological Technology, Jiangsu Province, China) according to the manufacturer's instructions, and the standard curve was $y=0.0028 x+0.3967\left(R^{2}=0.9987\right)$. CTK content was measured using the Plant CTK ELISA Kit (Jingmei Biological Technology, Jiangsu Province, China) according to the manufacturer's instructions, and the standard curve was $\mathrm{y}=0.0119 \mathrm{x}+0.0126\left(\mathrm{R}^{2}=0.9916\right)$. IAA content was measured using the Plant IAA ELISA Kit (Jingmei Biological Technology, Jiangsu Province, China) according to the manufacturer's instructions, and the standard curve was $y=0.0101 x+0.0263\left(R^{2}=0.9944\right)$. ETH content was measured using the Plant IAA ELISA Kit (Jingmei Biological Technology, Jiangsu Province, China) according to the manufacturer's instructions, and the standard curve was $y=0.0106 x+0.013\left(R^{2}=0.9989\right)$. 


\section{Extraction and measurement of key metabolites and enzymes in glycolysis (EMP) pathway and tricarboxylic acid (TCA) cycle}

Glucose was extracted following the methods described by Wang (Wang et al. 2012), pyruvate was extracted following Pandey (Pandey et al. 2002), acetyl CoA was extracted following the methods of Yang (Yang and Wu. 2013), hexokinase extraction was based on the methods of Schaffer (Schaffer and Petreikov, 1997), phosphofructokinase extraction was based on Singla (Singla et al. 2003), pyruvate kinase was extracted based on the methods of Wang (Wang et al. 2014), and citrate synthase was extracted following Tong (Tong et al. 2009). The Plant Glucose, Pyruvate, Acetyl CoA, Hexokinase, Phosphofructokinase, Pyruvate kinase, Citrate synthase ELISA kits (Jingmei Biological Technology, Jiangsu Province, China) were used respectively for the detection of the above metabolites/enzymes according to the manufacturer's instructions. The standard curve of glucose was $y=0.45 x+0.1008\left(R^{2}=0.9925\right)$. The standard curve of pyruvate was $y=0.0149 x+0.0014\left(R^{2}=0.9997\right)$. The standard curve of acetyl CoA was $y=0.005 x+0.039$ $\left(R^{2}=0.9952\right)$. The standard curve of hexokinase was $y=0.025 x-0.0411\left(R^{2}=0.9959\right)$. The standard curve of phosphofructokinase was $\mathrm{y}=0.0091 \mathrm{x}+0.0018$ $\left(\mathrm{R}^{2}=0.9974\right)$. And the standard curve of pyruvate kinase was $y=0.0021 x+0.005\left(R^{2}=0.9971\right)$. The standard curve of citrate synthase was $y=0.0005 x+0.0119\left(R^{2}=0.999\right)$.

\section{Extraction and measurement of energy metabolism and redox-related substances}

The extraction of ATP was performed following the method of Tang (Tang et al. 2003), and the Plant ATP ELISA kit (Jingmei Biological Technology, Jiangsu Province, China) was used for detection. The standard curve was $y=0.003 x+0.0644\left(R^{2}=0.9999\right)$. NADH, NAD ${ }^{+}$, $\mathrm{NADPH}$ and $\mathrm{NADP}^{+}$were ground and extracted with phosphate-buffered saline. NADH/NAD ${ }^{+}$was detected by $\mathrm{NAD}^{+} / \mathrm{NADH}$ assay kits (WST-8 method) (Beyotime Biotechnology, Shanghai, China), and the standard curve was $\mathrm{y}=56.589 \mathrm{x}+0.0698\left(\mathrm{R}^{2}=0.9994\right) . \mathrm{NADPH} / \mathrm{NADP}^{+}$was detected by NADP ${ }^{+} / \mathrm{NADPH}$ assay kits (WST- 8 method) (Beyotime Biotechnology, Shanghai, China), and the standard curve was $y=0.3218 x+0.0863\left(R^{2}=0.9996\right)$.

\section{Extraction of total RNA and quantitative real-time PCR}

The TRIzol method was used to extract total RNA (RNA isolater Total RNA Extraction Reagent) (Vazyme, Nanjing, China). The RNA was reverse-transcribed into cDNA (Hiscript II Q RT Supermix for qPCR (+gDNA wiper)) (Vazyme, Nanjing, China). Fluorescence quantitative realtime PCR (AceQ Universal SYBR qPCR Master Mix) (Vazyme, Nanjing, China) was performed, with $E F 1-\alpha$ as the reference gene, to determine the relative expression of the target genes. The primers sequences were designed using Premier 5.0 and Oligo 7. The primer sequences are shown in Table 1. The relative expression levels of genes were calculated by the $2^{-\Delta \Delta \mathrm{Ct}}$ method.

\section{Data analysis}

Each experiment in this study was repeated three times, with at least six biological repeats. The results are shown as the average value and standard error of six samples, calculated using SPSS 17.0 (SPSS Inc., Chicago, USA). For comparisons of parameters between wild-type and mutant plants, $t$-test were used (Wang et al. 2021).

\section{Results}

\section{Comparison of growth parameters}

We have previously found a mutant $A$. lancea tissue culture seedling with early stem growth, and better growth than that of wild-type tissue culture seedlings (Fig. 1), including larger leaves and more developed roots. The A. lancea mutant has a higher sesquiterpenoid content, which is closely related to improved rhizome development. At 3 months, the
Table 1 Quantitative realtime PCR primers for sesquiterpenoid synthesis related genes

\begin{tabular}{llll}
\hline Gene & $\begin{array}{l}\text { GenBank } \\
\text { accession } \\
\text { numbers }\end{array}$ & Forward primer $\left(5^{\prime}-3^{\prime}\right)$ & Reverse primer $\left(5^{\prime}-3^{\prime}\right)$ \\
\hline$E F 1-\alpha$ & X97131 & CAGGCTGATTGTGCTGTTCTTA & TGTGGCATCCATCTTGT \\
$H M G R$ & EF090602.1 & GGTGAGAAAGGTCCTGAAA & CATGGTAACGGAGATATGAA \\
$D X R$ & KF991105 & AAGATGTTAAAGTAGCCGATGC & GAATGTATGATAGAATGAGGGT \\
$N E S$ & OK398118 & AGTGGGTCAGCAACTGTCGT & GATTTATATTTGTGAGGAAGGCCAT \\
$C P S$ & MK694951 & GTAGCACTTCCCTTTGGTTT & TGTCGTTCCCTTGTTTGGCAGACAT \\
$G A S$ & OK398116 & TTGAGTCAGTGCGTCCTTTA & TAGTATTAGCTGCATCACTGGCCAT \\
\hline
\end{tabular}



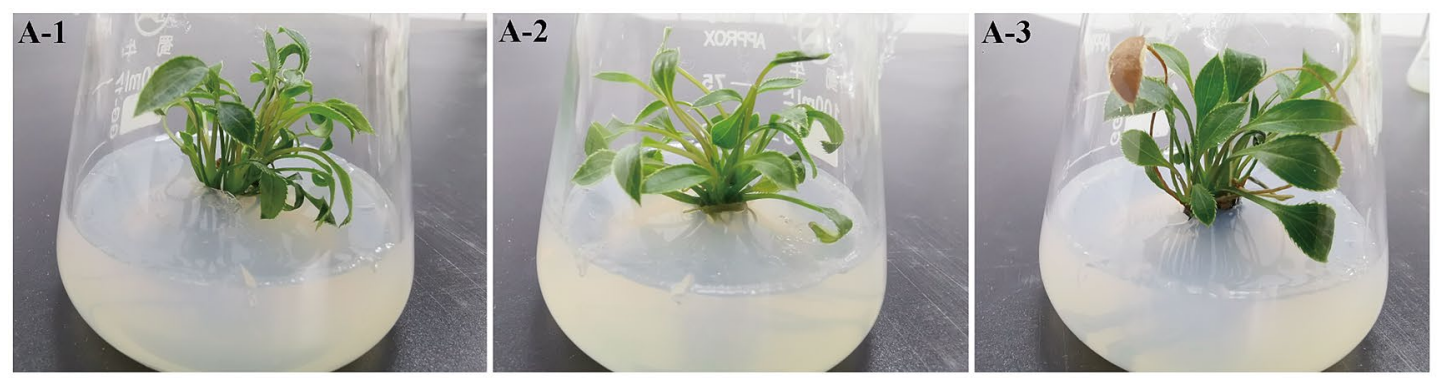

wt shoot
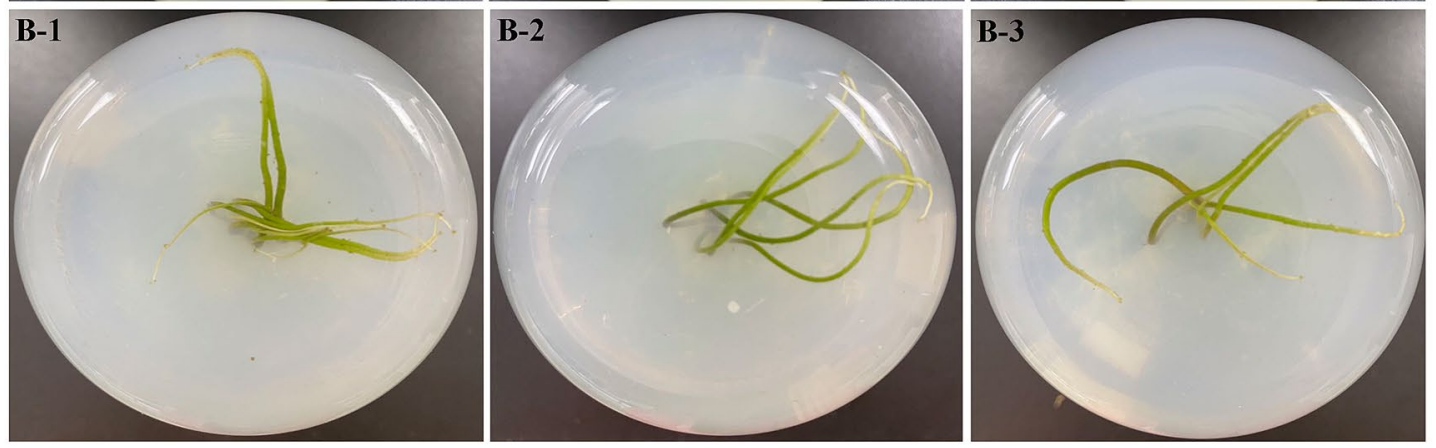

wt root
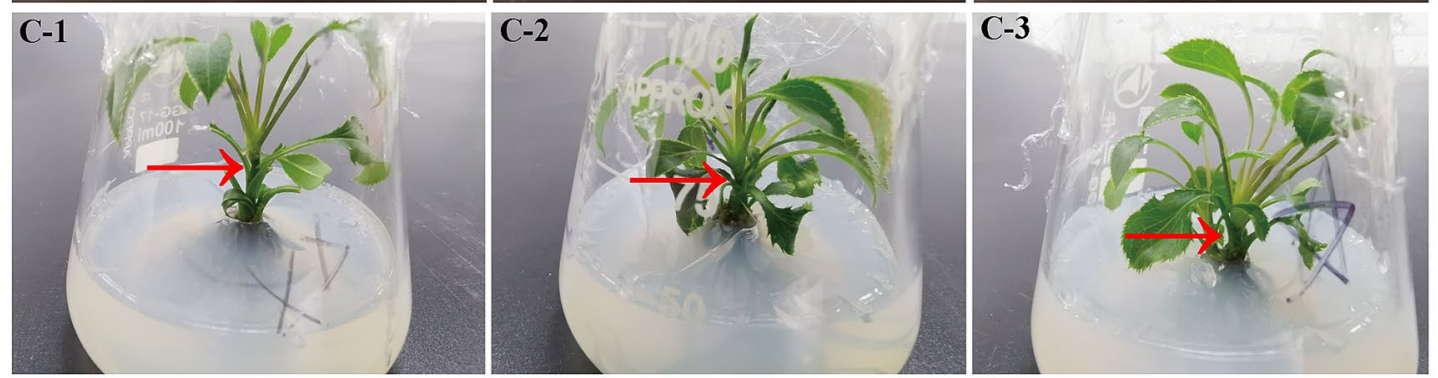

mt shoot
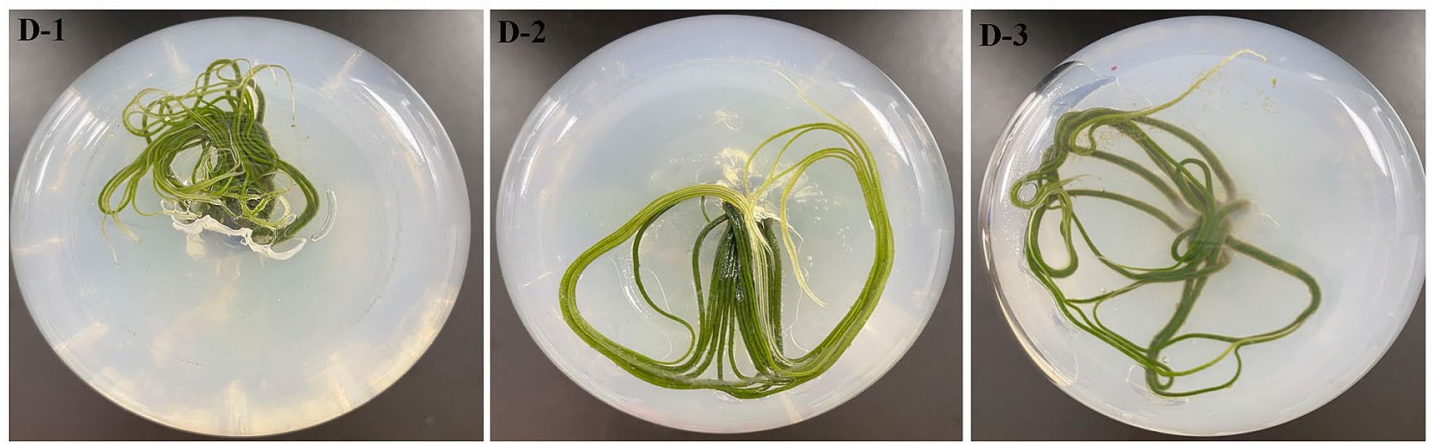

mt root

Fig. 1 Comparison between wild type and mutant A. lancea tissue culture seedlings. The culture time was 3 months. A-1-A-3 The shoots of wild type, B-1-B-3 the roots of wild type, C-1-C-3 the shoots of mutant type, D-1-D-3 the roots of mutant type. wt: wild type; mt: mutant type. The red scissor point is the advanced development of stem

of mutant plants was higher than that of wild-type at all time points, with significant and highly significant differences at 3 and 6 months. The number of leaves was always significantly higher in mutant $A$. lancea than in wild-type plants. These results clearly indicate that the the growth of the mutant is better than that of wild type.

Organic compounds produced by photosynthesis contribute substantially to plant growth and development. For a comparison of photosynthesis, we measured the chlorophyll content and the maximum photochemical quantum yield of narrow space of the tissue culture bottles. The fresh weight 
Table 2 Agronomic characters of wild-type and mutant A. lancea

\begin{tabular}{llllccc}
\hline Time (months) & Type & Fresh weight $(\mathrm{g})$ & Height $(\mathrm{cm})$ & Number of blades & Root length $(\mathrm{cm})$ & Number of roots \\
\hline 1 & Mutant & $0.30 \pm 0.07$ & $3.5 \pm 0.15$ & $17 \pm 2.64^{* *}$ & $3.0 \pm 0.55$ & $8 \pm 1.15$ \\
& Wild & $0.22 \pm 0.03$ & $3.7 \pm 0.51$ & $8 \pm 1.73$ & $2.9 \pm 0.10$ & $7 \pm 3.00$ \\
2 & Mutant & $0.81 \pm 0.39$ & $4.1 \pm 0.38$ & $27 \pm 5.29^{*}$ & $5.4 \pm 1.27$ & $14 \pm 6.24$ \\
& Wild & $0.39 \pm 0.09$ & $3.8 \pm 0.26$ & $15 \pm 3.51$ & $4.9 \pm 0.62$ & $12 \pm 0.00$ \\
3 & Mutant & $1.15 \pm 0.20^{*}$ & $5.0 \pm 0.55$ & $26 \pm 11.93^{*}$ & $8.0 \pm 1.91$ & $10 \pm 3.21$ \\
6 & Wild & $0.52 \pm 0.15$ & $5.0 \pm 0.55$ & $9 \pm 1.73$ & $5.4 \pm 0.99$ & $32 \pm 13.11$ \\
& Mutant & $2.04 \pm 0.12^{* *}$ & $5.4 \pm 0.30$ & $26 \pm 4.04^{*}$ & $9.9 \pm 0.45$ & $7.9 \pm 1.03$ \\
\hline
\end{tabular}

The calculation results are from the average of the six plants. The value before \pm is the mean, and the value after \pm is the standard errors, *Indicates that there is a significant difference $(\mathrm{P}<0.05)$, **Indicates that there is a very significant difference $(\mathrm{P}<0.01)$

photosystem II (Fv/Fm) in the wild-type and mutant plants (Fig. 2). The chlorophyll a and b contents increased initially and decreased thereafter, reaching peak at 2 months. The chlorophyll contents of mutant plants were always higher than those of wild type, and the difference was significant at 3 months (Fig. 2A). Fv/Fm did not change substantially during the 6-month period, indicating that the primary light energy conversion efficiency of the photosystem II reaction center of leaves remained basically constant. The efficiency of the mutant plants was slightly higher than that of the wild-type plants (Fig. 2B), although the difference was not statistically significant.

\section{Comparison of sesquiterpenoid contents}

In this study, 12 sesquiterpenoids in wild-type and mutant $A$. lancea were detected, including the oxygenated sesquiterpenoids atractylone, $\beta$-eudesmol, hinesol, caryophyllene oxide, cedrol, and nerolidol, and the oxygen-free sesquiterpenoids farnesene, germacrene $\mathrm{D}, \beta$-caryophyllene, zingiberene, and $\beta$-sesquiphellandrene. Sesquiterpenoids with high contents in shoot were mainly $\beta$-sesquiphellandrene, $\beta$-eudesmol and atractylone, and those with high contents in root were mainly $\beta$-sesquiphellandrene, atractylone, and farnesene.

Levels of each component showed an increasing trend, and the synthesis and accumulation of sesquiterpenoids were more efficient in mutant than in wild-type A. lancea, especially in root. Interestingly, in the wild-type tissue culture seedlings, the total content of these 12 sesquiterpenoids in shoot was higher than that in root, while the total content of these 12 sesquiterpenoids in root was higher than that in shoot in the mutant tissue culture seedlings (Fig. 3), because the roots of mutant grew faster and generated sesquiterpenoids more efficiently. The sesquiterpenoid content is closely related to the degree of root development. The roots of mutant A. lancea grew faster, with earlier transformation of the stem into rhizome (Fig. S1). There are oil chambers in the rhizome that stores abundant volatile oils. In the mutant type, oil chambers formed after 6 months of tissue culture and 6 months of pot culture, with no oil chamber formation
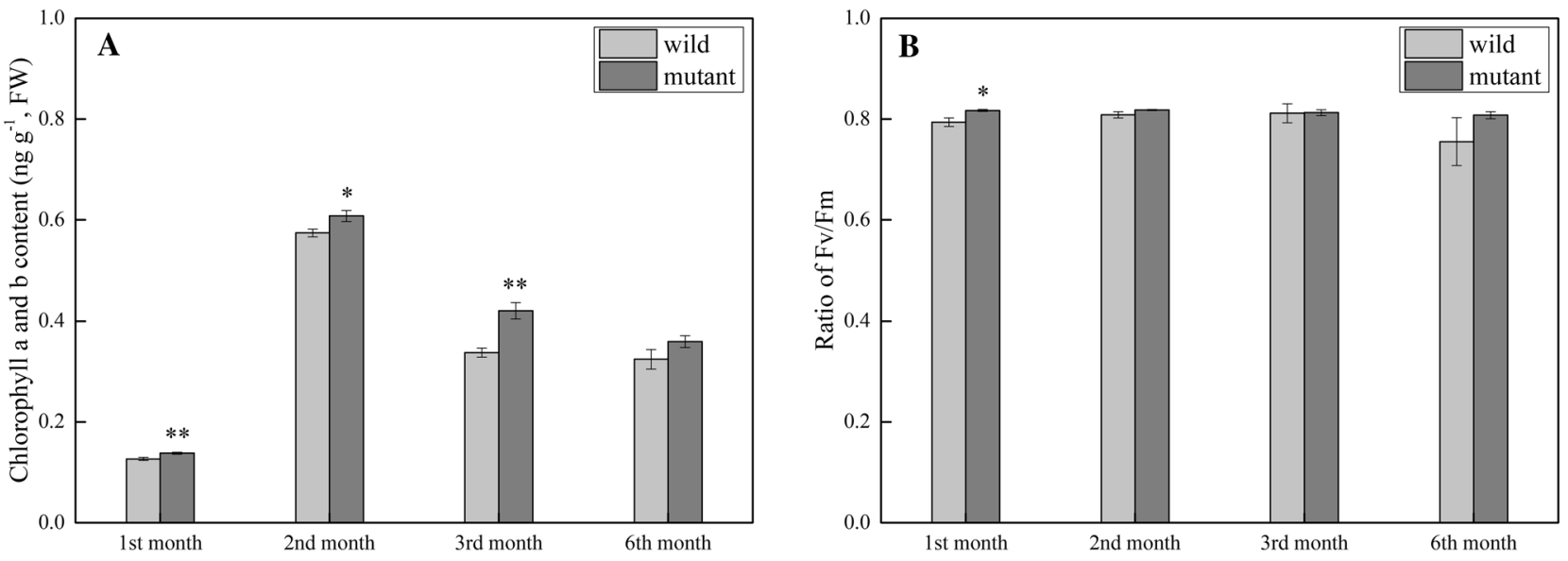

Fig. 2 Photosynthetic intensity of A. lancea. A The chlorophyll contents. B The maximum photochemical quantum yield of PS II. *Indicates that there is a significant difference $(\mathrm{P}<0.05), * *$ Indicates that there is a very significant difference $(\mathrm{P}<0.01)$ 

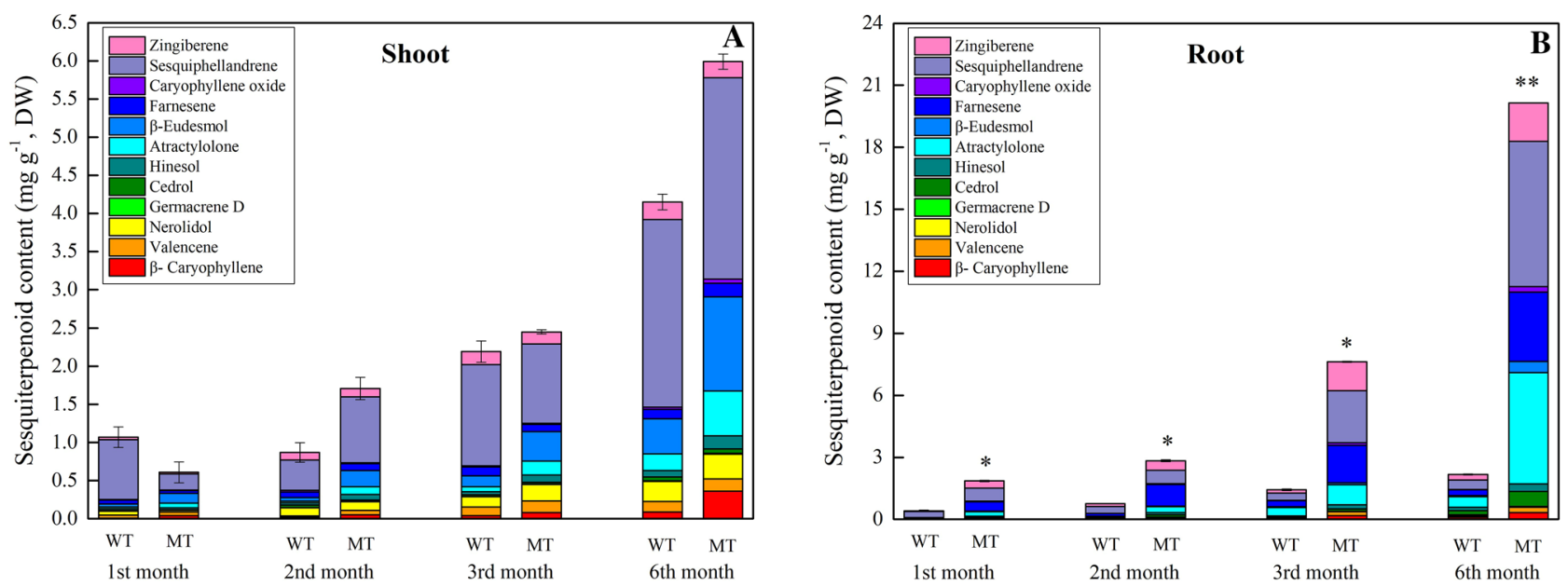

Fig. 3 Sesquiterpenoid contents in shoot and root of wild-type and mutant-type A. lancea. A Sesquiterpenoid contents in shoot. B Sesquiterpenoid contents in root

in the wild type at this time point (Fig. S2). The oil chamber provides a basis for evaluating the accumulation of sesquiterpenoid content in A. lancea (Hiraoka 1995), the early oil chamber formation in mutant $A$. lancea indicates that more sesquiterpenoids could accumulate.

In addition, the total content of oxygen-free sesquiterpenoids was always higher than that of oxygenated sesquiterpenoids in both mutant and wild-type plants, and both shoots and roots (Fig. S3). At present, there are two views on the formation of oxygenated sesquiterpenoids: (1) they are formed by the natural oxidation of basic sesquiterpenoids, and (2) they are formed by specific enzymes (Li et al. 2021). No matter which of the two viewpoints, it can be concluded that oxygen-free sesquiterpenoids were first biosynthesized, and oxygenated sesquiterpenoids were further formed from oxygen-free sesquiterpenoids. As shown in Fig. S3, compared with that in the wild type, although the contents of oxygen-free and oxygenated sesquiterpenoids increased in mutant type, the content of oxygenated sesquiterpenoids increased more obviously. This means that the advantage of the mutant in sesquiterpenoid synthesis is mainly reflected in the formation of oxygen-free sesquiterpenoids. According to our previous studies, oxygen-free sesquiterpenoids, as the basic sesquiterpenoids of A. lancea, were generally formed through primary metabolic pathway to form precursor substances, and further catalyzed by sesquiterpene synthase (Chen et al. 2017). Hereafter this text will prove these mainly from the perspective of primary metabolism.

\section{Comparison of endogenous hormone changes}

The superior agronomic characteristics of mutant A. lancea can be explained by many factors, including plant hormones, which contribute to plant growth and secondary metabolism.
$\mathrm{GA}$ is a kind of tetracyclic diterpenoid plant hormone, with an important role in the regulation of plant growth and development, including the effects on plant height, nutrient absorption, seed dormancy and germination (Gao and Chu. 2020). During A. lancea growth, the GA content increased continuously and was higher in mutant than in wild-type plants (Fig. 4A). Additionally, the temporal trend in plant height was the same as the trend in GA contents. However, ethylene did not differ significantly between wild-type and mutant plants (Fig. 4B).

The overall change in the cytokinin (CTK) content was roughly consistent with the changes in chlorophylls $\mathrm{a}$ and $\mathrm{b}$ (Fig. 2A), the content was the highest in the first month and lowest in the sixth month. Moreover, the CTK content of the mutant plants was significantly higher than that of the wildtype plants (Fig. 4D), indicating that the rate of cell division was higher in the of mutant type. Indoleacetic acid (IAA) is a ubiquitous endogenous hormone in plants, also known as auxin. In the early growth stage, the IAA content in mutant plants was higher than in wild-type plants. However, as the culture time increased, the IAA content tended to be similar in the two plant types (Fig. 4C), which may be explained by the depletion of nutrients in the culture medium.

Although the SA content was higher in wild-type plants than in mutant type in the second and third month, it exhibited an increasing trend in the mutant plants and a decreasing trend in the wild type. The SA content of the mutant type exceeded that of wild-type plants in the sixth month (Fig. 4F). JA contributes to biological and abiotic stress responses, and is very important for plant stress resistance (Chen et al. 2021). Additionally, JA participates in the signaling pathway for mediating sesquiterpenoid synthesis (Ren and Dai. 2012). JA contents decreased in 

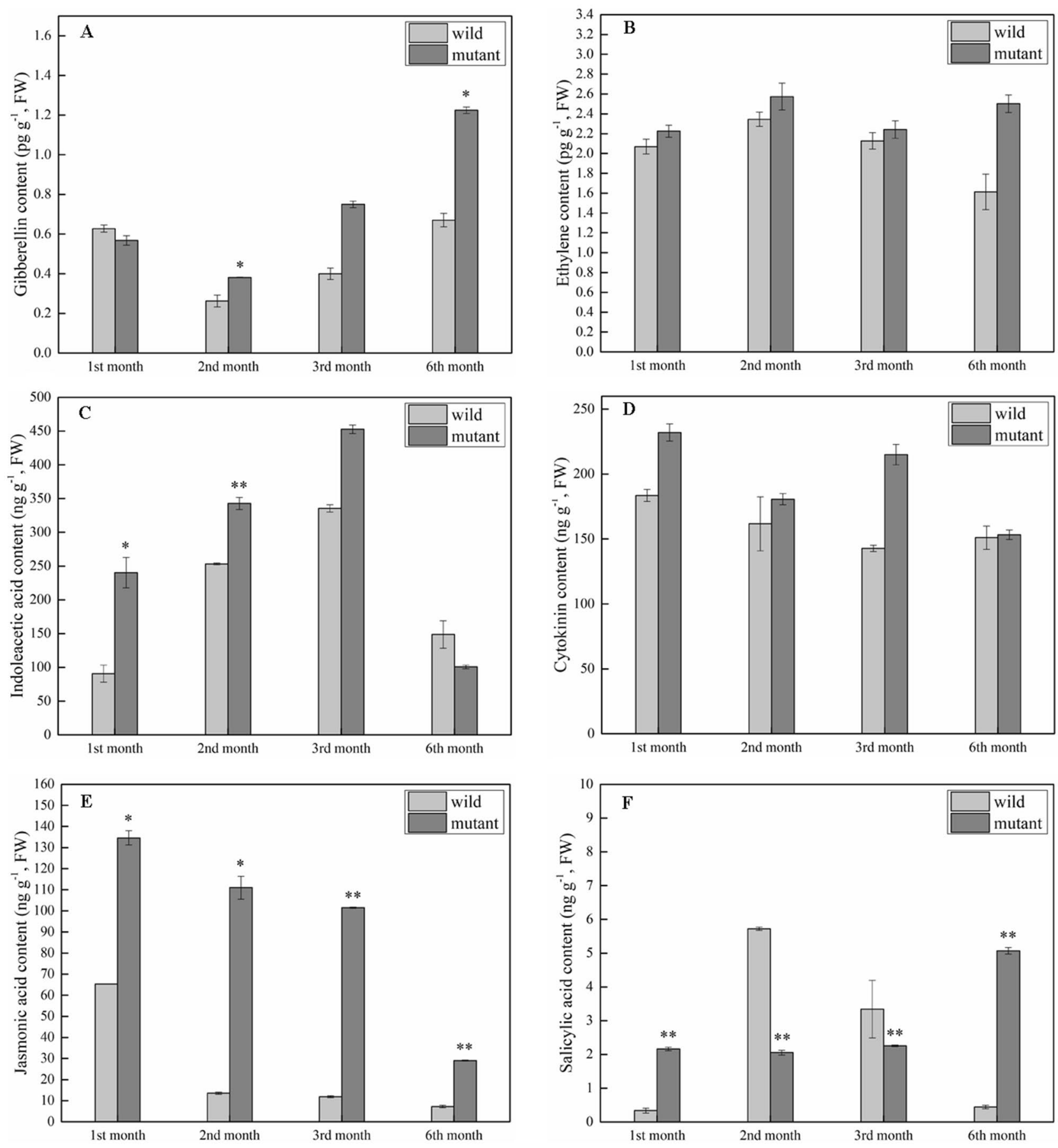

Fig. 4 Plant hormone content of A. lancea. A Gibberellin content. B Ethylene content. C Indoleacetic acid content. D Cytokinin content. E Jasmonic acid content. F Salicylic acid content. *Indicates that there

both wild-type and mutant $A$. lancea, and were always significantly higher in the mutant than in the wild type (Fig. 4E). These results indicated that mutant $A$. lancea has better stress resistance and could accumulate more sesquiterpenoids. is a significant difference $(\mathrm{P}<0.05)$, **Indicates that there is a very significant difference $(\mathrm{P}<0.01)$

\section{Comparison of central carbon metabolic pathways}

Sesquiterpenoids are secondary metabolites of A. lancea. Primary metabolism is necessary to provide precursors for the synthesis of the sesquiterpenoid skeleton. As the core metabolic pathways of primary metabolism, the EMP 
pathway and TCA cycle provide precursors and energy for many reactions, and are indicators of the metabolic activity of plants.

Hexokinase catalyzes the synthesis of fructose-6-phosphate from glucose, and produces ATP. The glucose content
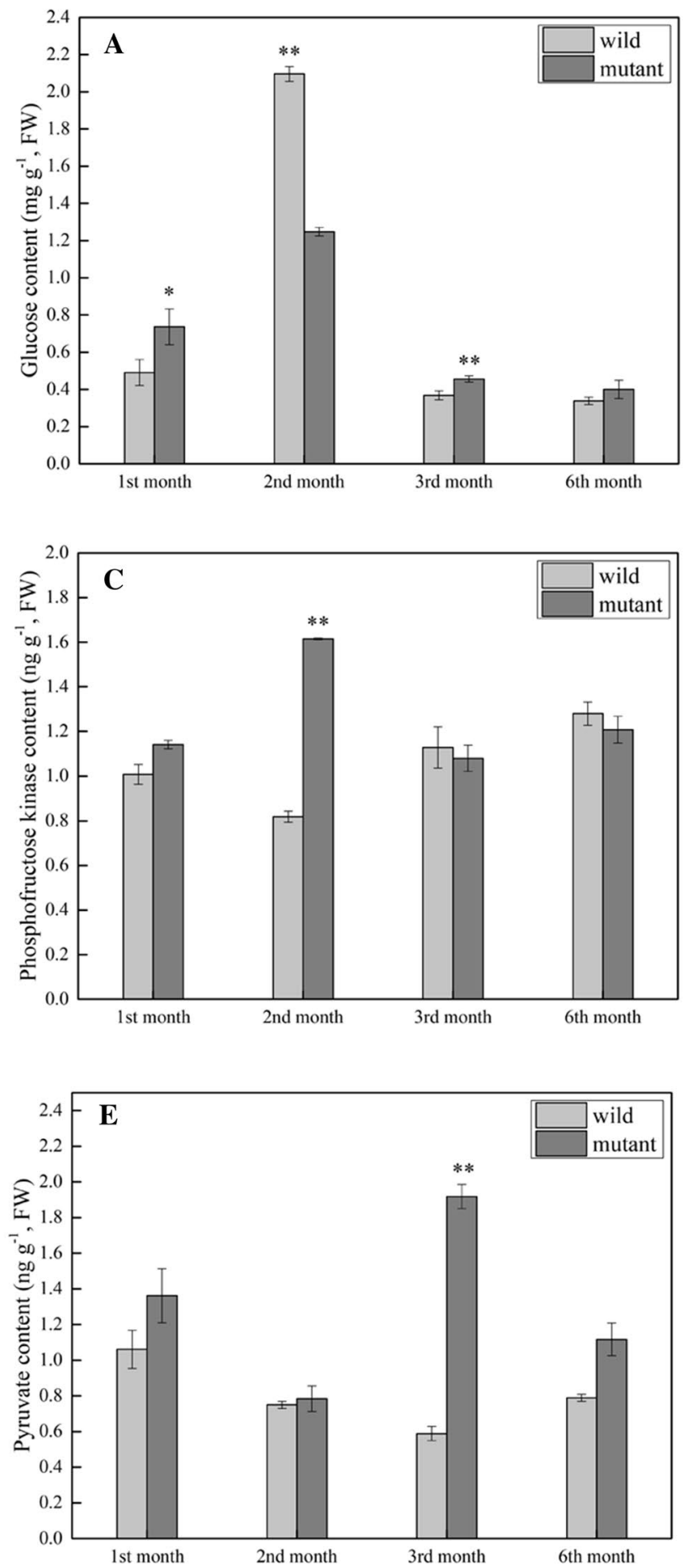

Fig. 5 Content of key enzymes and substances in EMP pathway. A Glucose content. B Hexokinase content. C Phosphofructose kinase content. D Pyruvate kinase content. E Pyruvate content. *Indicates showed an opposite trend to that of hexokinase content (Fig. 5B) in both wild-type and mutant plants. The glucose content reached the peak at 2 months, and then started to decreased substantially (Fig. 5A). Tissue culture seedlings absorbed glucose from the culture medium, and did not
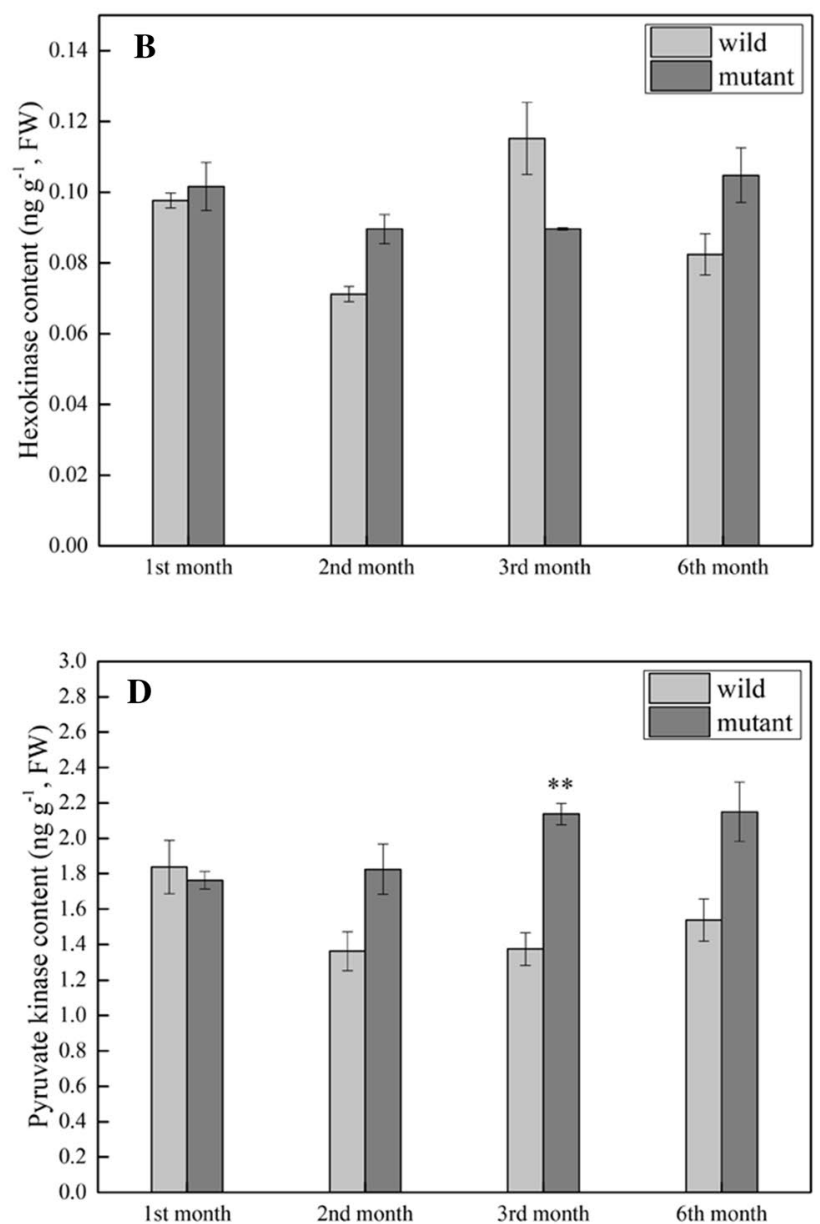

significant difference $(\mathrm{P}<0.05), * *$ Indicates that there is a very significant difference $(\mathrm{P}<0.01)$ 
consume large quantities. When the growth condition was relatively stable in 3 months, plants began to use the stored glucose.

The reactions catalyzed by phosphofructokinase and pyruvate kinase are also important steps in EMP pathway. In the first and second month, the phosphofructokinase of mutant type was higher in the mutant than in the wild type with similar contents for culture times of 3 months and 6 months (Fig. 5C). Pyruvate kinase catalyzes phosphoenolpyruvate to synthesize pyruvate, an important precursor of 2-C-methyl-D-erythritol-4-phosphate (MEP) pathway, able to synthesize acetyl CoA by the pyruvate dehydrogenase system. The changes in pyruvate and pyruvate kinase contents were generally similar. Both reached a peak in the third month of culture, with significant differences between the wild-type and mutant plants (Fig. 5D). The mutant type had an advantage in EMP pathway, as evidenced by the increase in fructose phosphate kinase activity in 2-month tissue culture seedlings and the increases in pyruvate kinase activity and the pyruvate content in 3-month tissue culture seedlings. Different from the changes in other ratelimiting enzymes, the pyruvate kinase increased gradually. After 3 months of culture, the secondary metabolic activity increased and sesquiterpenoid synthesis was more active, requiring more pyruvate. Therefore, pyruvate kinase was upregulated to meet the pyruvate demand for sesquiterpenoid synthesis, with peak levels in the third month of tissue culture (Fig. 5E). At 6 months, the main carbon source, sucrose, in the culture medium was consumed (Fig. S4), and there were few differences between the wild type and mutant type.

Tricarboxylic acid (TCA) cycle is the hub of metabolic relationships among carbohydrates, lipids, and amino acids. Acetyl CoA participates in the TCA cycle and is a precursor of the MVA pathway. Levels of acetyl CoA and citrate synthase showed the opposite trends (Fig. 6). When the culture time was 3 months, the activity of citrate synthase is the highest, and a large amount of acetyl CoA is consumed. The citrate synthase content in mutant plants was always higher than wild-type plants. As the only rate-limiting enzyme in the TCA cycle, an improvement in citrate synthase activity will increase the efficiency of the TCA cycle. Therefore, the growth, development, and metabolic rate of mutant plants are higher than those of wild-type plants, conferring advantageous of agronomic characters. Moreover, the TCA cycle will produce $\mathrm{NADH}+\mathrm{H}^{+}$, entering the respiratory chain and producing energy. The increase in TCA cycle activity is expected to substantially improve biological activity in the plant. Acetyl CoA is not only the substrate of citrate synthase, but also the precursor of the MVA pathway. The increase in the acetyl CoA content will provide additional precursors for MVA pathway, resulting in greater sesquiterpenoid accumulation.

\section{Comparison of energy substances and reducing activity}

ATP provides energy for many biological activities. For a culture time of 3 months, the ATP content reached a peak, and was two-fold higher in the mutant than in wild-type plants, indicating greater metabolic activity in the mutant (Fig. 7A). $\mathrm{NADH}_{2}$ releases a large amount of energy through the respiratory chain, as evaluated by the ratio of NADH to $\mathrm{NAD}^{+}$. This ratio was consistently higher in the mutant type than in the wild type, and the difference was significant in the third month (Fig. 7B). Sesquiterpenoid synthesis of $A$. lancea is an energy-intensive process, and the mutant has beneficial energy-related traits.

3-hydroxy-3-methylglutaryl CoA synthase (HMGR) and 1-deoxy-D-xylulose-5-phosphate reductoisomerase (DXR) enzymatic reactions require NADPH to supply hydrogen in

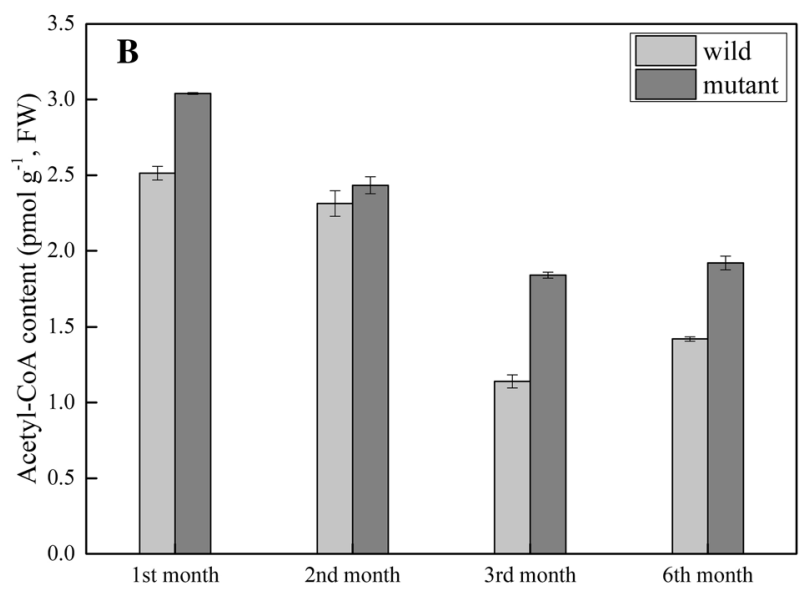

Fig. 6 Contents of key enzymes and substances in TCA cycle. A Citric acid synthase content. B Acetyl CoA content. *Indicates that there is a significant difference $(\mathrm{P}<0.05)$ 

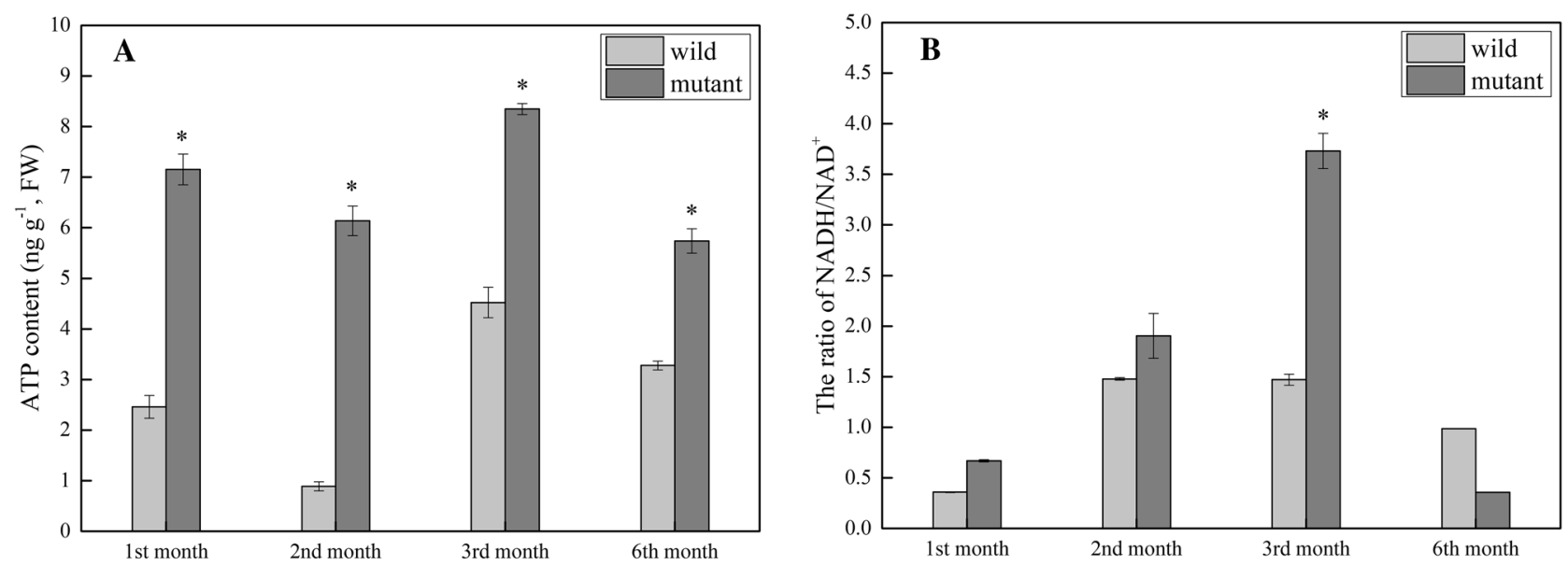

Fig. 7 Energy related substances. A ATP content. B The ratio of NADH to $\mathrm{NAD}^{+}$. * indicates that there is a significant difference $(\mathrm{P}<0.05)$

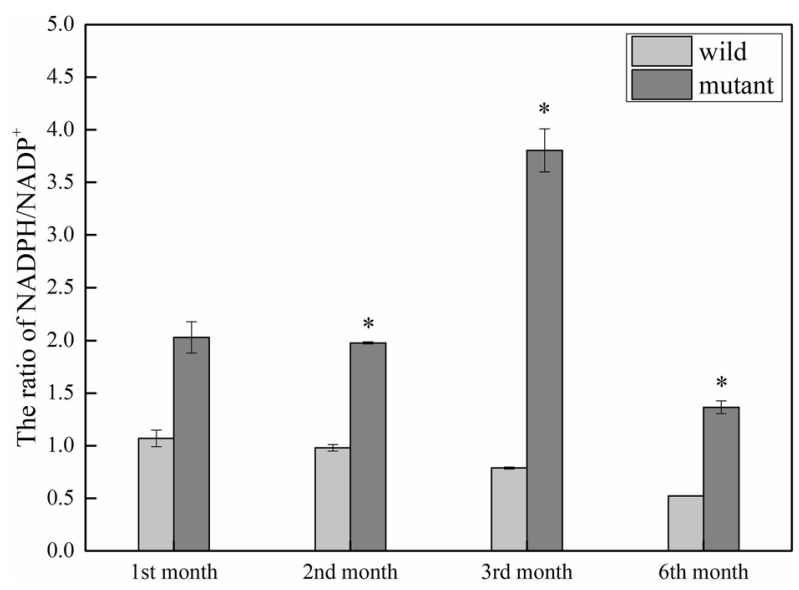

Fig. 8 Ratio of NADPH to NADP ${ }^{+}$. *Indicates that there is significant difference $(\mathrm{P}<0.05)$

the MVA pathway and EMP pathway. The ratio of NADPH to $\mathrm{NADP}^{+}$is an indicator of the reducing power of plants, where a larger value indicates a stronger reducing power. In the initial stage of growth, the reduction advantage of the mutant type was not obvious. In the process of sesquiterpenoid accumulation, a reduction advantage of the mutant type was gradually detected, especially at 3 months (Fig. 8).

\section{Expression levels of sesquiterpenoid synthesis-related genes in A. Iancea}

Sesquiterpenoid synthase of $A$. lancea controls the synthesis of sesquiterpenoids. Based on transcriptome data for $A$. lancea, we identified HMGR,DXR, AlCPS ( $\beta$-caryophyllene synthase), AlGAS (germacrene A synthase) and AlNES (nerolidol synthase) as critical genes for the synthesis of sesquiterpenoids (Chen et al. 2017). Therefore, we evaluated the expression levels of these genes in shoot and root (Fig. 9). The expression levels of $H M G R$ and DXR decreased over time in the aboveground plant part. These two genes control the MVA pathway and MEP pathway. Consistent with this, the expression levels were high in the early growth stage. When sufficient upstream products accumulated, the expression levels decreased, and at this time, A. lancea tends to synthesize more sesquiterpenoids through the expression of sesquiterpene synthase genes (i.e., AlCPS, AlGAS, AlNES).

$A l G A S$ levels were higher in the mutant than in the wild type, except in shoot in the first and the third month. The expression of AlGAS in shoot of the mutant type was highest at 3 months, and was significantly higher than that of the wild type. No $A l G A S$ expression was detected in root at 1 and 6 months. The expression of AlNES was higher in the mutant than in the wild type, except in root at 1 and 2 months. The trend in AlNES expression in shoot was similar to the trend in AlGAS expression. The expression of AlNES in root was highest at 3 months. The expression of AlCPS in shoot showed an overall upward trend, and was always higher in the mutant than in the wild type. The expression of AlCPS was not detected in root. Overall, the expression levels of genes related to sesquiterpenoid synthase were higher in the mutant than in the wild type at most time points, consistent with the results for volatile oil content detected by GC.

\section{Discussion}

Plant metabolic processes are mainly divided into primary and secondary metabolism. Primary metabolites are abundant and provide precursors for secondary metabolism. There are few secondary metabolites, however, they have important ecological functions, such as resistance to diseases and pests (Dhyani et al. 2021). Our results revealed 

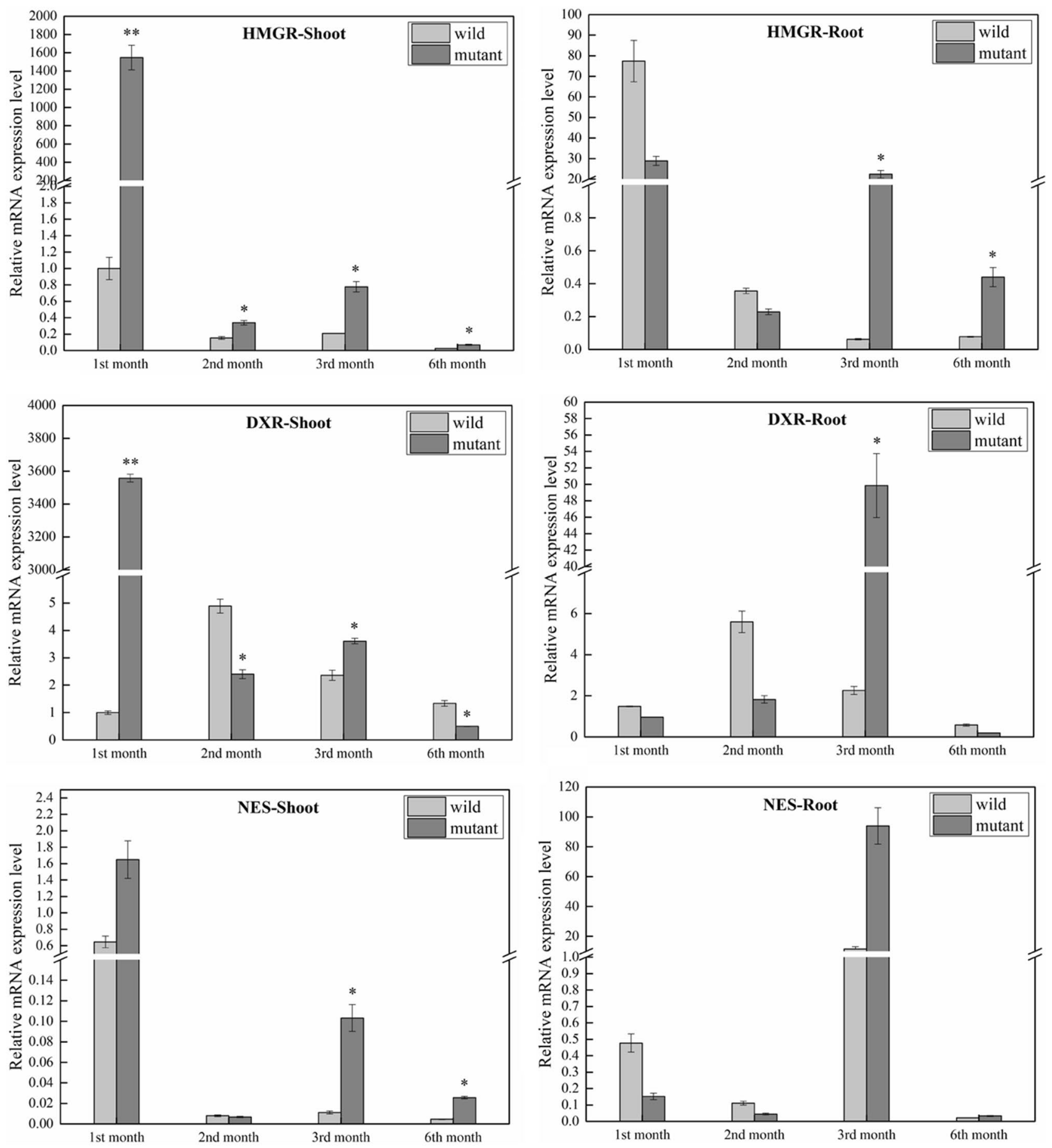

Fig. 9 Expression of sesquiterpenoid synthesis related genes in $A$. lancea. The relative expression levels of the target genes were calculated for the internal reference gene EFI- $\alpha$. *Indicates that there is a

that better accumulation of secondary sesquiterpenoids in mutant $A$. lancea can be explained by the primary metabolic pathway, and signaling pathways mediated by endogenous hormones (Fig. 10). In view of the core positions of EMP pathway and TCA cycle in primary metabolism, we significant difference $(\mathrm{P}<0.05), * *$ Indicates that there is a very significant difference $(\mathrm{P}<0.01)$

evaluated the changes of key enzymes and metabolites. We also measured the intensity of photosynthesis as well as the contents of plant hormones related to growth and sesquiterpenoid synthesis. The expression levels of sesquiterpenoid synthesis related genes were also measured. 

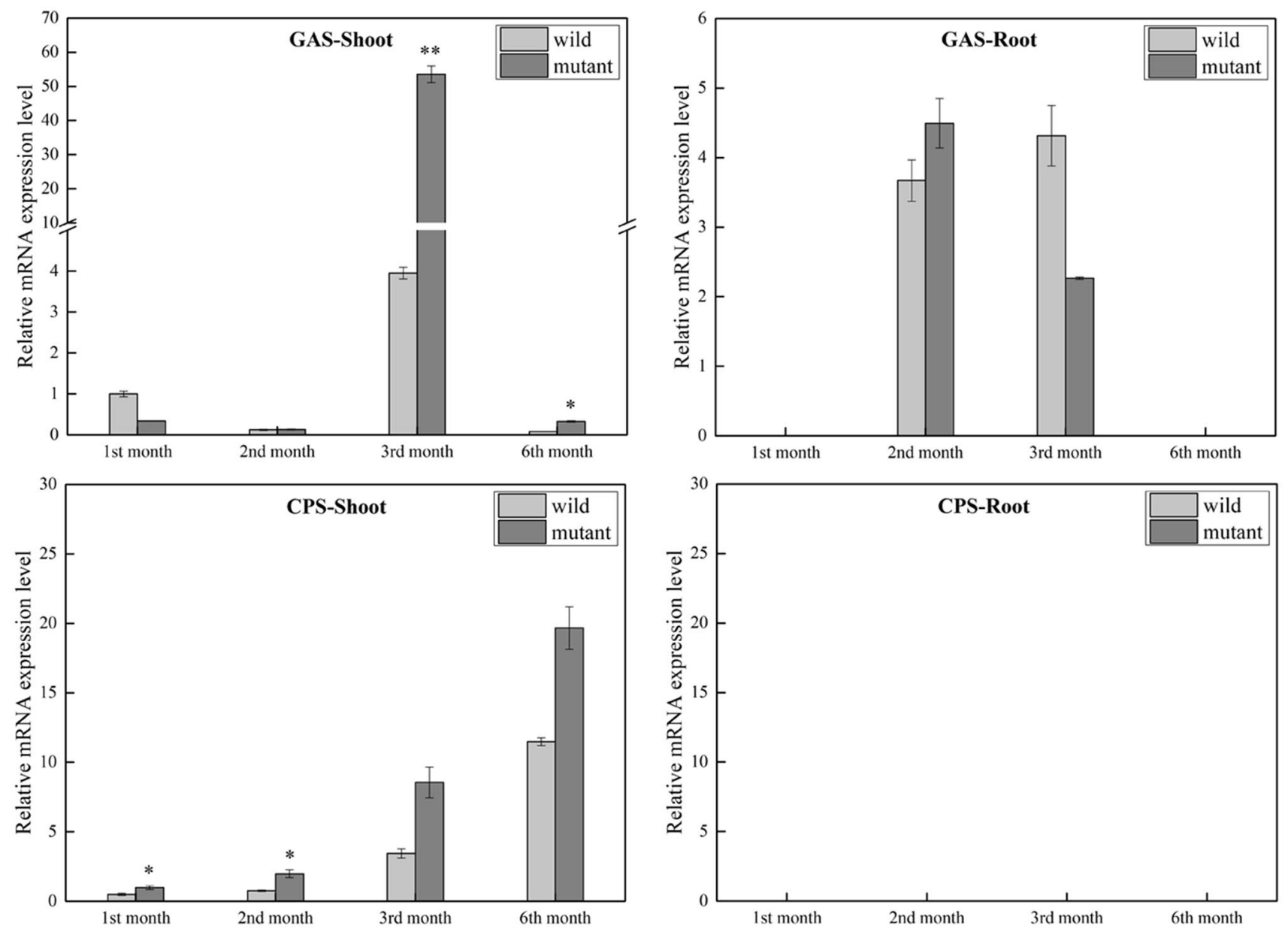

Fig. 9 (continued)

Photosynthesis provides materials for growth and development. It can convert inorganic carbon into available organic carbon. The chlorophyll content is closely related to the intensity of plant photosynthesis. In our study, the chlorophyll a and $\mathrm{b}$ contents as well as the primary light energy conversion efficiency of the photosystem II reaction center were similar in mutant and wild-type plants. However, isopentenyl diphosphate (IPP) and dimethylallyl diphosphate (DMAPP) are common precursors of sesquiterpenoids and chlorophylls ( $\mathrm{Zi}$ et al. 2014), mutant A. lancea used more IPP and DMAPP to synthesize sesquiterpenoids. Therefore, we speculate that the mutant A. lancea is more likely to synthesize more sesquiterpenoids than to consume excessive "material resources" to improve the efficiency of photosynthesis.

Plant hormones are often involved in plant biological activities, including plant biosynthesis, metabolism, perception, and signal transduction. Changes in plant hormone signaling clearly contribute to the accelerated growth and the increased accumulation of secondary metabolites in mutant A. lancea. Previous studies have shown that GA is involved in the regulation of many agronomically important traits, including plant height, nutrient use efficiency, seed germination, and tolerance to various abiotic and biological stresses (Gao and Chu. 2020). CTK can induce cell division, accelerate chlorophyll synthesis and delay leaf senescence. It is often regarded as an indicator of chloroplast biosynthesis, which reflects the intensity of plant photosynthesis to a certain extent (Cortleven et al. 2016). At the cellular level, IAA can stimulate the division of cambium cells and regulate callus morphogenesis. The signaling pathways mediated by JA, SA, and ETH were up-regulated to various degrees, which could induce greater accumulation of volatile oils in A. lancea (Ren et al. 2017; Ren and Dai. 2012, 2013). Therefore, we speculate that the changes of plant hormone contents have an important impact on the advantageous properties of mutant A. lancea. In particular, the GA and JA contents increased significantly in the mutant. GA influences plant height, stem development and internode elongation, explaining the increase in the height of mutant plants. The increase in the GA content was positively correlated with plant height (Table 2, Fig. 4A, Fig. S5). JA regulates many physiological 


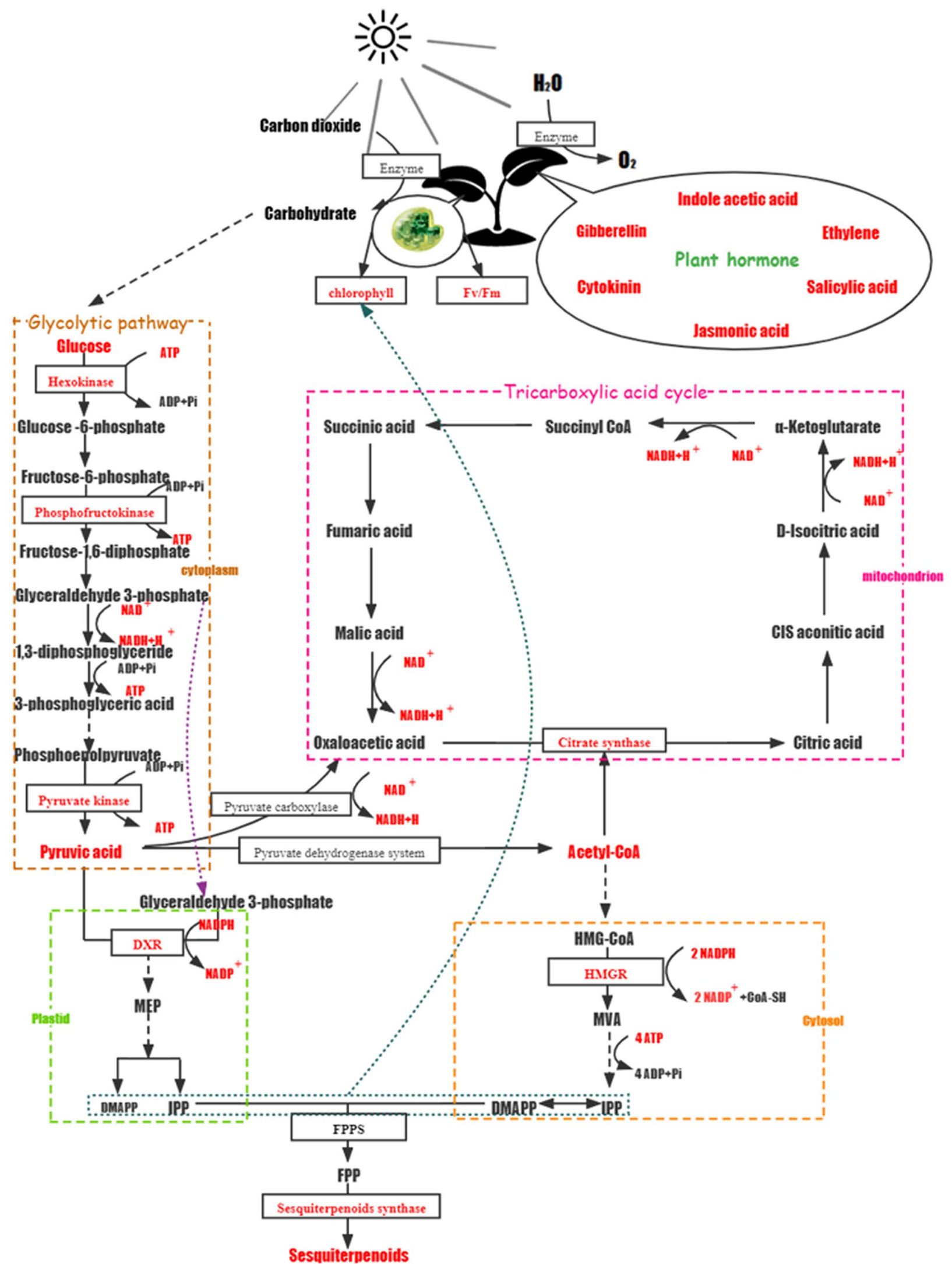


४Fig. 10 Schematic diagram of primary metabolism and sesquiterpenoid synthesis related pathways of A. lancea. Refered to previous literatures for drawing (Maeda. 2019; Liu et al. 2016). The substances marked in red are measured in this research, including photosynthetic efficiency, central carbon metabolism efficiency, energy metabolism efficiency, plant hormone levels, and gene expression levels

processes and the response to changes in many abiotic factors, such as light, temperature, humidity, salt, $\mathrm{CO}_{2}$, and $\mathrm{O}_{3}$ (Ruan et al. 2019). JA also protects against biological stresses. It mediates the accumulation of sesquiterpenoids via $\mathrm{H}_{2} \mathrm{O}_{2}$ ( $\mathrm{Zi}$ et al. 2014). There was an antagonistic relationship between SA and JA, reflected in the second and third month. Therefore, we speculate that the increase in the GA content promotes growth and development, and the increase in the JA content promotes the accumulation of sesquiterpenoids in mutant $A$. lancea. The up-regulation of genes encoding key enzymes in MVA and MEP pathways further supports the increase in sesquiterpenoids accumulation in mutant A. lancea.

Our experimental data show that the advantages of mutant A. lancea are largely reflected in an improvement of energy metabolism. Both the ATP content and NADH/NAD ${ }^{+}$ratio were consistently higher in the mutant than in the wild type. ATP is involved in almost all energy-consuming reactions, and NADH releases a lot of energy when entering the respiratory chain, providing a basis for improved sesquiterpenoid synthesis and biological processes in mutant $A$. lancea. The MVA and MEP pathways are key steps in plant sesquiterpenoid synthesis, occurring in the cytosol and plastids, respectively (Maeda. 2019). These two pathways require not only energy sources, but also reducing power (hydrogen), mainly from NADPH. The ratio of NADPH to $\mathrm{NADP}^{+}$in mutant $A$. lancea was always higher than that in wild-type A. lancea, indicating stronger reducing power. The contents of pyruvate and acetyl CoA in mutant $A$. lancea were also increased. These factors will improve the intensity of HMGR and DXR catalytic reactions, thereby improving the efficiency of sesquiterpenoids synthesis.

Sesquiterpenoids are synthesized mainly via the MVA and MEP pathways (Nagata et al. 2002). IPP and DMAPP are common precursors of terpenoids. The sesquiterpenoid precursor FPP is synthesized by catalysis of FPPS (Nagegowda. 2010), and then processed into corresponding sesquiterpenoids by various sesquiterpenoid synthases. Metabolic regulatory enzymes usually contribute to post-translational regulation (Hemmerlin. 2013). IPP and DMAPP is a complex and tightly regulated progress. They are not only used to synthesize terpenoids (monoterpenes, sesquiterpenoids and triterpenes) in secondary metabolism (Maeda. 2019), but are also important precursors in the synthesis of photosynthetic pigments. Furthermore, they also synthesize some plant hormones such as GA, SA and BR (Zi et al. 2014). IPP and DMAPP play important roles in MVA and MEP pathways. MVA and MEP are catalyzed by HMGR and DXR, respectively, and sterols, sesquiterpenoids, and triterpenes are further synthesized by a series of enzymes. In plants, the MEP pathway functions in plastids, DXR is the key enzyme in this pathway. However, mutant $A$. lancea did not show obvious differences in this pathway. Similarly, photosynthesis in the plastid did not increase significantly in the mutant A. lancea. Accordingly, we speculate that the plastid of mutant A. lancea was not altered substantially. The MVA pathway mainly functions in the cytosol. HMGR is a key enzyme of this pathway, while the downstream reaction catalyzed by PMK and MDC occurs in the peroxisome (Maeda 2019). The expression of $H M G R$ in the mutant was always higher than that in the wild type. The increase in $H M G R$ expression level has an important impact on the efficiency of sesquiterpenoid synthesis. In view of advanced development of the oil chamber of mutant A. lancea and the increase in the sesquiterpenoid content, we speculated that the improvement in the efficiency of the sesquiterpenoid synthesis pathway catalyzed by HMGR is an important determinant of the elevated sesquiterpenoid content of the mutant.

Based on our physiological and biochemical analysis, we conclude that the advantageous properties of mutant A. lancea (i.e., increased plant height and sesquiterpenoid content) are caused by many factors, including increases in energy metabolism, reducing power, sesquiterpenoid precursors, and the efficiency improvement of signaling pathways related to sesquiterpenoids synthesis mediated by GA and JA (Fig. 10). In summary, the primary metabolic activity of mutant $A$. lancea was significantly enhanced. Although we focused on the physiological and biochemical mechanisms underlying traits in mutant $A$. lancea, regulatory changes at the molecular level, such as changes in epigenetic factors lead to regulation of key target genes for sesquiterpenoid synthesis, may also be critical. In the following studies, we will conduct microRNAs to regulate sesquiterpenoid biosynthesis through the morphogenesis of mutant A. lancea. Further explorations underlying the molecular mechanism of the advantageous properties of mutant A. lancea will improve our understanding of sesquiterpenoid synthesis and provide new directions for excellent germplasm resources breeding.

Supplementary Information The online version contains supplementary material available at https://doi.org/10.1007/s11240-022-02240-5.

Acknowledgements This work was financially supported by Key Project at Central Government Level: The ability establishment of sustainable use for valuable Chinese medicine resources (Grant No. 2060302), the National Natural Science Foundation of China (Grant No. 21406119), the Key Program of the National Natural Science Foundation of China (Grant No. 81891014), and a project funded by the Priority Academic Program Development (PAPD) of Jiangsu Higher Education Institutions. 
Author contributions FC conceived and designed the experiments. $\mathrm{C}-\mathrm{CD}$ made some constructive suggestions for the manuscript. DW, $\mathrm{C}-\mathrm{YW}, \mathrm{XH}$ and FC performed the experiments. DW and FC analyzed the data and wrote the manuscript.

\section{Declarations}

Conflict of interest Authors declare that they have no conflict of interest and the publication of the work has been approved by all coauthors.

Ethical approval No human participants and /or animals are involved in this work.

Consent for publication All the authors agree with the publication of the article.

\section{References}

Bessho-Uehara K, Nugroho JE, Kondo H, Angeles-Shim RB, Ashikari M (2018) Sucrose affects the developmental transition of rhizomes in Oryza longistaminata. J Plant Res 131:693-707

Chen F, Ren CG, Zhou T, Wei YJ, Dai CC (2016) A novel exopolysaccharide elicitor from endophytic fungus Gilmaniella sp. AL12 on volatile oils accumulation in Atractylodes lancea. Sci Rep 6:34735

Chen F, Wei YX, Zhang JM, Sang XM, Dai CC (2017) Transcriptomics analysis investigates sesquiterpenoids accumulation pattern in different tissues of Atractylodes lancea (Thunb.) DC. plantlet. Plant Cell Tissue Organ Cult 130:73-90

Chen CL, Yuan F, Li XY, Ma RC, Xie H (2021) Jasmonic acid and ethylene signaling pathways participate in the defense response of Chinese cabbage to Pectobacterium carotovorum infection. $\mathrm{J}$ Integr Agr 20:1314-1326

Cortleven A, Marg I, Yamburenko MV, Schlicke H, Hill K, Grimm B, Schmulling T (2016) Cytokinin regulates the etioplast-chloroplast transition through the two-component signaling system and activation of chloroplast-related genes. Plant Physiol 172:464-478

Dhyani R, Srivastava SK, Shankar K, Ghosh T, Beniwal A, Navani NK (2021) A chemical genetic approach using genetically encoded reporters to detect and assess the toxicity of plant secondary metabolites against bacterial pathogens. J Hazard 418:126399

Gao S, Chu C (2020) Gibberellin metabolism and signaling: targets for improving agronomic performance of crops. Plant Cell Physiol 61:1902-1911

Guo L, Plunkert M, Luo X, Liu Z (2021) Developmental regulation of stolon and rhizome. Curr Opin Plant Biol 59:101970

Hemmerlin A (2013) Post-translational events and modifications regulating plant enzymes involved in isoprenoid precursor biosynthesis. Plant Sci 203-204:41-54

Hiraoka N (1995) Intra-Plant distribution of essential oil components oil accumulation tissues in Atractylodes lancea. Nat Med 49:168-171

Hu F, Wang D, Zhao X, Zhang T, Sun H, Zhu L, Li Z (2011) Identification of rhizome-specific genes by genome-wide differential expression analysis in Oryza longistaminata. BMC Plant Biol $11: 18$

Jun X, Fu P, Lei Y, Cheng P (2018) Pharmacological effects of medicinal components of Atractylodes lancea (Thunb.) DC. Chin Med $13: 59$

Li HY, Canagaratna MR, Riva M, Rantala P, Zhang YJ, Thomas S, Bianchi F (2021) Atmospheric organic vapors in two European pine forests measured by a Vocus PTR-TOF: insights into monoterpene and sesquiterpene oxidation processes. Atmos Chem Phys 21:4123-4147

Liu Y, Wang Q, Ding Y, Li G, Xu J, Wang S (2011) Effects of external ABA, GA3 and NAA on the tiller bud outgrowth of rice is related to changes in endogenous hormones. Plant Growth Regul 65:247-254

Liu Q, Zhang S, Yang X, Wang R, Guo W, Kong W, Yang M (2016) Differentiation of essential oils in Atractylodes lancea and Atractylodes koreana by gas chromatography with mass spectrometry. J Sep Sci 39:4773-4780

Liu J, Yang W, Liu Y et al (2021) Combination of Hua Shi Bai Du granule (Q-14) and standard care in the treatment of patients with coronavirus disease 2019 (COVID-19): a single-center, open-label, randomized controlled trial. Phytomedicine 91:153671

López-Bucio J, Cruz-Ramírez A, Herrera-Estrella L (2003) The role of nutrient availability in regulating root architecture. Curr Opin Plant Biol 6:280-287

Maeda HA (2019) Harnessing evolutionary diversification of primary metabolism for plant synthetic biology. J Biol Chem 294:16549-16566

Malamy JE (2005) Intrinsic and environmental response pathways that regulate root system architecture. Plant Cell Environ 28:67-77

Nagata N, Suzuki M, Yoshida S, Muranaka T (2002) Mevalonic acid partially restores chloroplast and etioplast development in Arabidopsis lacking the non-mevalonate pathway. Planta 216:345-350

Nagegowda DA (2010) Plant volatile terpenoid metabolism: biosynthetic genes, transcriptional regulation and subcellular compartmentation. FEBS Lett 584:2965-2973

Pandey DM, Goswami CL, Kumar B, Jain S (2002) Effect of growth regulators on photosynthetic metabolites in cotton under water stress. Biol Plant 45:445-448

Patten CL, Glick BR (2002) Role of Pseudomonas putida indoleacetic acid in development of the host plant root system. Appl Environ Microbiol 68:3795-3801

Porra RJ (2002) The chequered history of the development and use of simultaneous equations for the accurate determination of chlorophylls a and b. Photosynth Res 73:149-156

Ren CG, Dai CC (2012) Jasmonic acid is involved in the signaling pathway for fungal endophyte-induced volatile oil accumulation of Atractylodes lancea plantlets. BMC Plant Biol 12:128

Ren CG, Dai CC (2013) Nitric oxide and brassinosteroids mediated fungal endophyte-induced volatile oil production through protein phosphorylation pathways in Atractylodes lancea plantlets. J Integr Plant Biol 55:1136-1146

Ren CG, Chen F, Dai CC (2017) Fungal endophyte protects Atractylodes lancea from root rot caused by Fusarium oxysporum. Plant Pathol 66:223-229

Ruan J, Zhou Y, Zhou M, Yan J, Khurshid M, Weng W, Zhang K (2019) Jasmonic acid signaling pathway in plants. Int J Mol Sci 20:2479

Schaffer AA, Petreikov M (1997) Inhibition of fructokinase and sucrose synthase by cytosolic levels of fructose in young tomato fruit undergoing transient starch synthesis. Physiol Plant 101:800-806

Singla NK, Jain V, Jain S, Sawhney SK (2003) Activities of glycolytic enzymes in leaves and roots of contrasting cultivars of sorghum during flooding. Biol Plant 47:555-560

Tang W, Brady SR, Sun Y, Muday GK, Roux SJ (2003) Extracellular ATP inhibits root gravitropism at concentrations that inhibit polar auxin transport. Plant Physiol 131:147-154

Tang H, Hu YY, Yu WW, Song LL, Wu JS (2015) Growth, photosynthetic and physiological responses of Torreya grandis seedlings to varied light environments. Trees 29:1011-1022

Tong J, Zhan GM, Wang XF, Liu GH, Hua W, Wang HZ (2009) Cloning of citrate synthase gene in rapeseed (Brassica napus L.) and its expression under stresses. Acta Agron Sin 35:33-40 
Wang Y, Dai CC, Cao JL, Xu DS (2012) Comparison of the effects of fungal endophyte Gilmaniella sp. and its elicitor on Atractylodes lancea plantlets. World J Microbiol Biotechnol 28:575-584

Wang XN, Zhao L, Li C, Ren Q, Liu XL (2014) Effects of different wall breaking methods on pyruvate kinase activity. Food Drugs $16: 257-260$

Wang XM, Yang B, Ren CG, Wang HW, Wang JY, Dai CC (2015) Involvement of abscisic acid and salicylic acid in signal cascade regulating bacterial endophyte-induced volatile oil biosynthesis in plantlets of Atractylodes lancea. Physiol Plant 153:30-42

Wang K, Gao E, Liu D, Wu X, Wang P (2021) The ER network, peroxisomes and actin cytoskeleton exhibit dramatic alterations during somatic embryogenesis of cultured citrus cells. Plant Cell Tissue Organ Cult. https://doi.org/10.1007/s11240-021-02180-6

Xu J, Chen D, Liu C, Wu XZ, Dong CX, Zhou J (2016) Structural characterization and anti-tumor effects of an inulin-type fructan from Atractylodes chinensis. Int J Biol Macromol 82:765-771

Yang Y, Wu GF (2013) Determination of acetyl coenzyme A in grape fruit by high performance liquid chromatography tandem mass spectrometry. Chromatography 31:33-37

Yang X, Zhang X, Yuan D, Jin F, Zhang Y, Xu J (2012) Transcript profiling reveals complex auxin signaling pathway and transcription regulation involved in dedifferentiation and redifferentiation during somatic embryogenesis in cotton. BMC Plant Biol 12:110

Yuan J, Sun K, Den Wang MY, Dai CC (2016) The mechanism of ethylene signaling induced by endophytic fungus Gilmaniella sp.
AL12 mediating sesquiterpenoids biosynthesis in Atractylodes lancea. Front Plant Sci 7:361

Zhang WJ, Zhao ZY, Chang LK, Cao Y, Wang S, Kang CZ, Guo LP (2021) Atractylodis Rhizoma: a review of its traditional uses, phytochemistry, pharmacology, toxicology and quality control. J Ethnopharmacol 266:31

Zhou JY, Li X, Zhao D, Deng-Wang MY, Dai CC (2016) Reactive oxygen species and hormone signaling cascades in endophytic bacterium induced essential oil accumulation in Atractylodes lancea. Planta 244:699-712

Zhou JY, Sun K, Chen F, Yuan J, Li X, Dai CC (2018) Endophytic pseudomonas induces metabolic flux changes that enhance medicinal sesquiterpenoid accumulation in Atractylodes lancea. Plant Physiol Biochem 130:473-481

Zi J, Mafu S, Peters RJ (2014) To gibberellins and beyond! Surveying the evolution of (di)terpenoid metabolism. Annu Rev Plant Biol 65:259-286

Publisher's Note Springer Nature remains neutral with regard to jurisdictional claims in published maps and institutional affiliations. 